2. R. C. Bose and W. H. Clatworthy, Partially balanced designs with two associate classes and $k>r=3, \lambda_{1}=1, \lambda_{2}=0$, in preparation for publication.

3. R. C. Bose and K. R. Nair, Partially balanced incomplete block designs, Sankhyā vol. 4 (1939) pp. 337-372.

4. R. C. Bose and T. Shimamoto, Classification and analysis of partially balanced incomplete block designs with two associate classes, Journal of the American Statistical Association vol. 47 (1952) pp. 151-184.

5. K. R. Nair and C. R. Rao, A note on partially balanced incomplete block designs, Science and Culture vol. 7 (1942) pp. 568-569.

University of North Carolina

\title{
SOME CONDITIONS FOR UNIFORM CONVERGENCE OF INTEGRALS
}

\section{EMANUEL PARZEN ${ }^{1}$}

Let $m$ be a sigma-finite measure on a space $R$, let $T$ be an abstract index set, and for each $t \in T$ and $n=1,2, \cdots$, let $f_{n}(x, t), f(x, t)$ be functions measurable in $x \in R$. It is of some interest to determine conditions for statements of the form

$$
\int_{R}\left|f_{n}(x, t)-f(x, t)\right| d m \rightarrow 0 \quad \text { uniformly in } t,
$$

where we use the conventions that if a limit is taken as $n \rightarrow \infty$, or if $t$ varies in $T$, then these facts will not be explicitly stated. Graves $[1$, p. 241, Theorem 22] states conditions for (1) to hold under the assumption that:

(2) at each $x \in R$, except for $x$ in a null set which is independent of $t, f_{n}(x, t)$ converges to $f(x, t)$ uniformly in $t$.

The mode of convergence defined by (2) is much too restrictive. In this paper we define modes of uniform convergence of a family of sequences of measurable functions which are much less restrictive than (2) and under which (1) holds. Situations of this more general kind were encountered by the author in [2], and the theorems proved below are there applied.

Before introducing our modes of convergence, let us recall that, given measurable functions $f, f_{n}(n=1,2, \cdots)$, Egorov's theorem states that $f_{n}(x) \rightarrow f(x)$ almost everywhere (that is, $m\left\{x: f_{n}(x)\right.$ does not converge to $f(x)\}=0$ ) if, and only if, for every $\epsilon>0$ and every

Presented to the Society, September 4, 1953; received by the editors March 30, 1953 and, in revised form, June 1, 1953.

1 National Science Foundation predoctoral fellow, 1952-53. Now at Columbia University, Hudson Laboratories, Dobbs Ferry, New York. 
measurable set $A$ of finite measure, as $N \rightarrow \infty, m\left\{x \in A:\left|f_{n}(x)-f(x)\right|\right.$ $>\epsilon$ for some $n>N\} \rightarrow 0$. Egorov's theorem thus suggests that there are two ways in which we might define almost everywhere convergence uniformly in $t$. The uniformity can be in the convergence of the functions themselves, which is the case in (2), or it can be in the convergence to zero of the measure of exceptional sets of nonconvergence. We therefore make the following definitions.

Definition I. $f_{n}(x, t) \rightarrow f(x, t)$ almost everywhere m-uniformly in $t$ if, for every $\epsilon>0$ and every measurable set $A$ of finite measure, as $N \rightarrow \infty$,

$$
m\left\{x \in A:\left|f_{n}(x, t)-f(x, t)\right|>\epsilon \text { for some } n>N\right\} \rightarrow 0
$$

uniformly in $t$.

Definition II. $f_{n}(x, t) \rightarrow f(x, t)$ in measure $m$-uniformly in $t$ if, for every $\epsilon>0$ as $n \rightarrow \infty, m\left\{x:\left|f_{n}(x, t)-f(x, t)\right|>\epsilon\right\} \rightarrow 0$ uniformly in $t$.

Unless $R$ is of total finite measure, neither (I) nor (II) imply the other. A mode of uniform convergence implied by both (I) and (II) is given by:

(II') for every $\epsilon>0$ and every measurable set $A$ of finite measure, as $n \rightarrow \infty, m\left\{x \in A:\left|f_{n}(x, t)-f(x, t)\right|>\epsilon\right\} \rightarrow 0$ uniformly in $t$.

Most of the relations that hold in measure theory between convergence almost everywhere, convergence in measure, and convergence in mean continue to hold between (I), (II), and (1). We shall here explicitly indicate some of these relations. To state our basic result on integration we recall the following notions, where for brevity we use the term summable to denote what is sometimes called absolutely continuous and convergent, or continuous from above at 0 .

(A) Integrals $\int_{R} f(x, t) d m$ are summable uniformly in $t$ if, to every $\epsilon>0$, there is a measurable set $A$ of finite measure and an $\eta>0$ such that for any measurable set $B$ satisfying $m(A B)<\eta$ we have $\int_{B}|f(x, t)| d m<\epsilon$ for any $t$.

(B) Integrals $\int_{R} f_{n}(x, t) d m$ are summable uniformly in $t$ asymptotically in $n$ if, to every $\epsilon>0$, there is a measurable set $A$ of finite measure, an $\eta>0$, and an integer $N$ such that for any measurable set $B$ satisfying $m(A B)<\eta$ we have $\int_{B}\left|f_{n}(x, t)\right| d m<\epsilon$ for any $n>N$ and $t$.

Let, for $\epsilon>0, \quad J_{n}(t)=\int_{R}\left|f_{n}(x, t)-f(x, t)\right| d m$, and $E_{n}(\epsilon, t)$ $=\left\{x:\left|f_{n}(x, t)-f(x, t)\right|>\epsilon\right\}$.

TheOREM. (1) and (A) hold if, and only if, (II) and (B) hold if, and only if, (II') and (B) hold.

Proof. That (1) and (A) imply (II), (II'), and (B) follows by the 
inequalities, for any $\epsilon>0$ and measurable sets $A$ and $B$,

$$
\begin{gathered}
m\left(A E_{n}(\epsilon, t)\right) \leqq m\left(E_{n}(\epsilon, t)\right) \leqq J_{n}(t) / \epsilon, \\
\int_{B}\left|f_{n}(x, t)\right| d m \leqq \int_{B}|f(x, t)| d m+J_{n}(t) .
\end{gathered}
$$

To conclude the proof, it suffices to show that (II') and (B) imply (1) and (A). Let $\epsilon>0$. To $\epsilon$ choose $A, \eta, N$ in accord with (B). Let $\epsilon^{\prime}=\epsilon / m(A)$. By $\left(\mathrm{II}^{\prime}\right)$, choose $N^{\prime}>N$ so that $m\left(A E_{n}\left(\boldsymbol{\epsilon}^{\prime}, t\right)\right)<\eta / 2$ for any $n>N^{\prime}$ and $t$. Let $C_{k n}(t)=A-E_{k}\left(\epsilon^{\prime}, t\right)-E_{n}\left(\epsilon^{\prime}, t\right), B_{k n}(t)=R$ $-C_{k n}(t)$. Then, for any $t$ and $k, n>N^{\prime}$, it follows that $m\left(A B_{k n}(t)\right)<\eta$, and

$$
\begin{aligned}
\int_{R}\left|f_{k}(x, t)-f_{n}(x, t)\right| & d m \\
& \leqq 2 \epsilon^{\prime} m\left(C_{k n}(t)\right)+\int_{B k n(t)}\left|f_{k}(x, t)-f_{n}(x, t)\right| d m \\
& \leqq 2 \epsilon^{\prime} m(A)+2 \epsilon=4 \epsilon .
\end{aligned}
$$

It follows that, uniformly in $t$ and measurable sets $A, \int_{A} f_{n}(x, t) d m$ approaches a limit which we know to be $\int_{A} f(x, t) d m$. From this, we easily infer (1) and (A).

Since (I) implies $\left(\mathrm{II}^{\prime}\right)$, from the above theorem we immediately obtain what might be called a uniform bounded convergence theorem.

Theorem. If (I) and (B) hold, then (1) and (A) hold.

In the converse direction, we have the following lemma which follows by the inequality for any $\epsilon>0$ and measurable set $A$, $m\left\{x \in A:\left|f_{n}(x, t)-f(x, t)\right|>\epsilon\right.$ for some $\left.n>N\right\} \leqq \sum_{n>N} m\left(E_{n}(\epsilon, t)\right)$.

Lemma. If $\sum_{n} J_{n}(t)$ converges uniformly in $t$, then (I) holds.

There does not seem to be any satisfactory uniform analogue of the monotone convergence theorem which is not already included in the above theorems. Next, let us note a further extension of our results. The modes of convergence we have defined, and the theorems we have stated, retain their validity, with certain obvious modifications in the statements, if we consider on $R$ a family of sigma-finite measures $m_{t}$ together with a family of sequences of measurable functions $f_{n}(x, t)$.

REMARK. A different kind of theorem on uniform convergence of integrals is one involving Riemann-Stieltjes integrals. Let $R$ be a 
finite-dimensional Euclidean space. For each $t \in T$ and $n=1,2, \cdots$, let $F_{n}(x, t), F(x, t)$ be uniformly bounded monotone functions on $R$. Given a bounded continuous function $g(x)$, it is of interest to determine conditions under which

$$
\int_{R} g(x) d F_{n}(x, t) \rightarrow \int_{R} g(x) d F(x, t) \quad \text { uniformly in } t .
$$

One finds in Graves [1, pp. 287-290] sufficient conditions for (3) to hold. Since results of this kind have important applications in probability theory, in Chapter 1 of [2] we were led to define a mode of uniform convergence of $F_{n}(x, t)$ to $F(x, t)$ which permits us to give necessary and sufficient conditions for (3) to hold for every bounded continuous function $g(x)$.

\section{REFERENCES}

1. L. M. Graves, Theory of functions of real variables, New York, McGraw-Hill, 1946.

2. E. Parzen, On uniform convergence of families of sequences of random variables, University of California Publications in Statistics, vol. 2, 1954, to appear.

University of California, Berkeley 